\title{
Research on the Ultimate Value of Compound Function in Higher Mathematics
}

\author{
Long Wang ${ }^{1, a}$, Zhe Wang ${ }^{2, b}$ \\ ${ }^{1}$ Shanghai University of Political Science and Law, China \\ ${ }^{2}$ Edinburgh Napier University, UK \\ aemail:wanglong59@163.com, bemail:z.wang2@napier.ac.uk
}

Keywords: compound function; ultimate value; existence; computation.

\begin{abstract}
The problem of ultimate value of compound function is very complex, especially the research on judging whether the existence of ultimate value of compound function under the given conditions and how is the exact conditions that can affect the existence of ultimate value is not enough, but this is the most attractive research questions for math researchers. This paper discuss the ultimate value of compound functions regarding to its existence possibility in two dimensions of compound functions theory and three layers of ultimate theory, so as to effectively solve the computation problems of ultimate value of compound functions.
\end{abstract}

\section{Introduction}

The problem of ultimate value of compound function $f[g(x)]$ is very complex, because it should be firstly judged whether the $\lim f[g(x)]$ is existing or not, especially by using what kind of method to judge the existence of the ultimate value of the compound function, secondly is how to calculate the $\lim f[g(x)]$.so that, separate the compound function into two layers.

(1) the first layer: set $u=g(x)$, and further considerate the existence of $\lim u=\lim _{x \rightarrow x_{0}} g(x)$

(2) the second layer: set $y=f(u)$, and further considerate the existence of $\lim y=\lim f(u)$.

Based on the above settings and foundations, this paper will discuss the existence of $\lim f[g(x)]$ and the computation problems with the given conditions which growing from weak to strong.

\section{Under the situation of weak Conditions}

Suppose the ultimate value of $\lim _{x \rightarrow x_{0}} u=\lim _{x \rightarrow x_{0}} g(x)=u_{0}$ is existing. Andthe ultimate value of $\lim _{u \rightarrow u_{0}}=\lim _{u \rightarrow u_{0}} f(u)=A \quad$ is also existing, then the question is: 1) it the ultimate value of $\lim f[g(x)]$ existing or not?; (2) under the situation of the ultimate, value of $\lim f[g(x)]$ is existing, how to calculate the value of $\lim f[g(x)]$.The answer is: it is very easy to solve the second question, which the ultimate value is $\lim f[g(x)]=\lim _{u \rightarrow u_{0}} f(u)=A$.The reason is very simple, because the existing of $\lim f[g(x)]$ is true,so that it only need to concern when $\mathrm{x}$ is belongs to alignment $x_{1}, x_{2}, x_{3}, \cdots$ and $\mathrm{x}$ has the tendency to become ${ }^{x_{0}}$,correspondingly the $u$ belongs to alignment $u_{1}, u_{2}, u_{3}, \cdots$ and $u$ has the tendency to become ${ }^{u_{0}}$, obviously, this is the solution. It is equivalent to when $\forall \varepsilon>0, \exists \delta>0$, and $0<\left|x-x_{0}\right|<\delta$, there is $\left|u-u_{0}\right|<\eta \quad((\eta>0)$, so as to get the result of $|f[g(x)]-A|<\varepsilon$ is validate. 
(1) So now, the critical problem is to solve question 1 . we look at the first sample, set $y=\left[1-u^{8}\right]$,represent the integrate part of $\left(1-u^{8}\right)$, and further set $u=x \sin \frac{1}{x}$,obviously, there is $\lim _{x \rightarrow 0} u=\lim _{x \rightarrow 0} x \sin \frac{1}{x}=0 \quad \lim _{u \rightarrow 0} y=\lim _{u \rightarrow 0}\left[1-u^{8}\right]=0$ however, when $x \rightarrow 0, u=x \sin \frac{1}{x}$,it can take $x=\frac{1}{2 k \pi}(k=1,2,3, \cdots)$,then, $u=x \sin \frac{1}{x}$ can get zero by infinite times, but when $u=0, y=1$,so that the function $y=\left[1-u^{8}\right]=\left[1-\left(x \sin \frac{1}{x}\right)^{8}\right]$ when $x=\frac{1}{2 k \pi}$, then $y=1$, it means the function has sub-alignments tends to 1 ,on the other side, when $0<\left|x \sin \frac{1}{x}\right|<1$,then $y=0$,so that the ultimate value of $\lim _{u \rightarrow 0} y=\lim _{x \rightarrow 0}\left[1-\left(x \sin \frac{1}{x}\right)^{8}\right]=0$ tend to value0. Based on the interval column compactness theorem, the necessary and sufficient conditions of alignment $a_{n} \rightarrow a$ is the sub-alignment of $a_{n}$, which is $a_{n_{k}} \rightarrow a$ is knowable. $\lim y$ is not exist, Then the answer for question 1 is negative.

Critical Analysis : although theultimate value of $\lim _{x \rightarrow x_{0}} u=\lim _{x \rightarrow x_{0}} g(x)=u_{0} \quad \lim _{u \rightarrow u_{0}}=\lim _{u \rightarrow u_{0}} f(u)=A$ are both existing, but in the process of replace the $u=g(x)$, when $x \rightarrow 0$, it may change $u$ that within a valid range which makes the ultimate value of $\lim _{u \rightarrow u_{0}} f(u)=A$ is existing during the process of $u \rightarrow u_{0}$, it is shown in the upper case.it is in the replacement of $u=x \sin \frac{1}{x}$, when $x \rightarrow 0, u$ can get infinite times to be 0 , so that it can change the parameter range that makes $\lim y$ non-existing.

Based on the above analysis, we found, in order to make sure question (1) has the definite answer, it has two ways of solutions, first is complete the additional conditions, second is change the parameter range of. So that, the following questions is proposed and then sum up the regulations

$$
\begin{gathered}
\lim _{x \rightarrow x_{0}} u=\lim _{x \rightarrow x_{0}} g(x)=u_{0}, \lim _{x \rightarrow u_{0}} f(u)=L \text {,in which conditions then the ultimate value is } \\
\lim _{x \rightarrow x_{0}} f[g(x)]=L \text { ? }
\end{gathered}
$$

from the definition of ultimate value, set $\varepsilon>0$, then $\eta$ is existing, when $0<\left|u-u_{0}\right|<\left|g(x)-u_{0}\right|<\eta$, then

$$
|f(u)-L|=|f(g(x))-L|<\varepsilon \text { is true }
$$

because $^{\lim _{x \rightarrow x_{0}} g(x)=u_{0}}$, so $\varepsilon>0$, then $\delta>0$ existing, makes when $0<\left|x-x_{0}\right|<\delta$, there is

$$
\left|g(x)-u_{0}\right|<\eta
$$

$\lim _{x \rightarrow x_{0}} f[g(x)]=L$, is equal to when $0<\left|x-x_{0}\right|<\delta$, there is

$$
|f(g(x))-L|<\varepsilon \text { is true }
$$

it is to proof from (4) to (3), So that, in (3), set $u \neq u_{0}$, if $u=u_{0}$, then the in-equivalent

$$
|f(u)-L|<\varepsilon
$$

may not true (for example the situation of $u=0$ ), based on the upon example, there is exist the $\mathrm{x}$ to make the in-equivalent 


$$
0<\left|x-x_{0}\right|<\delta \text { is true }
$$

, then $u=g(x)=u_{0}$ (for example the $x=\frac{1}{2 k \pi}, k=1,2,3, \cdots, u=x \sin \frac{1}{x}=0$ ), but $|f(u)-L|<\varepsilon$ is not reasonable., so that, under the conditions for the given problems it is hard to guarantee the (3) can be proofed from (4),: so that the additional conditions is needed.,

I、 in (4) $g(x)=u_{0}$ there is no $x$ to make it true

II when $u=u_{0},|f(u)-L|<\varepsilon$ is true,

Based on the above analysis, the discipline for calculating the ultimate value of compound function is:

1 set $^{x \rightarrow x_{0}} \lim _{x \rightarrow x_{0}} g(x)=u_{0} \lim _{u \rightarrow u_{0}} f(u)=L$, when $x=x_{0} \quad$ (or exclude $x=x_{0}$ ) there is no $x$ makes $g(x)=u_{0}$, then

$$
\lim _{x \rightarrow x_{0}} f[g(x)]=\lim _{u \rightarrow u_{0}} f(u)=L
$$

1 based on $\lim _{x \rightarrow \frac{\pi}{2}} u=\lim _{x \rightarrow \frac{\pi}{2}} \cos x=0$, and $\lim _{u \rightarrow 0} \frac{\sin u}{u}=1$,in this example, when $x \rightarrow \frac{\pi}{2}$, there is no $x$ to make $\cos x=0$,based on rule1there is

$$
\lim _{x \rightarrow \frac{\pi}{2}} \frac{\sin (\cos x)}{\cos x}=\lim _{u \rightarrow 0} \frac{\sin u}{u}=1
$$

\section{Under the Strong conditions}

in the disciple 1 the conditions is $\lim _{x \rightarrow x_{0}} u=\lim _{x \rightarrow x_{0}} g(x)=u_{0}$,based on $\lim _{x \rightarrow u_{0}} f(u)=L$,set $f\left(u_{0}\right)=L$, because $f\left(u_{0}\right)=L$ effectively constrains the range of the variable $u$,or can say it exclude the situation when $x \rightarrow x_{0}$, during $u \rightarrow u_{0}$ it may change the range of $u$, which makes the $\lim _{u \rightarrow u_{0}} f(u)=A$ is existing,, : based on the above analysis:

$$
\text { set } \begin{gathered}
\lim _{x \rightarrow x_{0}} u=\lim _{x \rightarrow x_{0}} g(x)=u_{0} \\
\lim _{x \rightarrow u_{0}} f(u)=L \text {, and } f\left(u_{0}\right)=L \text {, then } \\
\lim _{x \rightarrow x_{0}} f(g(x))=\lim _{u \rightarrow u_{0}} f(u)
\end{gathered}
$$

example2 $\lim _{x \rightarrow 0}(1+x)^{\frac{1}{x}}=e \quad, \quad y=\ln (1+x)^{\frac{1}{x}} \quad$,because $y=\ln u \quad$ and $\quad f\left(u_{0}\right)=L$,set $\left.\ln u\right|_{u=e}=\ln e$,based on rule2

$$
\lim _{x \rightarrow 0} \ln (1+x)^{\frac{1}{x}}=\ln \left[\lim _{x \rightarrow 0}(1+x)^{\frac{1}{x}}\right]=\ln e=1
$$

example $3^{\lim _{\Delta x \rightarrow 0} \frac{\Delta y}{\Delta x}=y^{\prime} \lim _{x \rightarrow x_{0}} u=\lim _{x \rightarrow x_{0}} g(x)=u_{0} \lim _{x \rightarrow u_{0}} f(u)=L}$,and $f\left(u_{0}\right)=L$, based on rule 2

$$
\lim _{\Delta x \rightarrow 0} \sqrt{1+\left(\frac{\Delta y}{\Delta x}\right)^{2}}=\sqrt{1+\left(\lim _{\Delta x \rightarrow 0} \frac{\Delta y}{\Delta x}\right)^{2}}=\sqrt{1+\left(y^{\prime}\right)^{2}}
$$

So that, when there is a compound function $f(g(x))$, and $u=g(x)$, based on the character of continuous functions, it is very ease to get:

$$
f(u), u=u_{0} \text { is not continuous,or } f\left(u_{0}\right)_{\text {no definition,or }} f\left(u_{0}\right) \neq \lim _{u \rightarrow u_{0}} f(u) \text {; }
$$


rule 2 is suitable for $f(u)$, when $u=u_{0}$, the $g(x)$ is continuous at $x=x_{0}$ has no relation with question,only makes $\lim _{x \rightarrow x_{0}} g(x)$ existing is fine.

III Condition is very Strong

Under the rule2 $\lim _{x \rightarrow x_{0}} u=\lim _{x \rightarrow x_{0}} g(x)=u_{0} \lim _{x \rightarrow u_{0}} f(u)=L$, and based on $f\left(u_{0}\right)=L$ further add the conditon $u_{0}=g\left(x_{0}\right)$,so we get rule 3 :

Rule 3, set $\lim _{x \rightarrow x_{0}} u=\lim _{x \rightarrow x_{0}} g(x)=g\left(x_{0}\right)=u_{0} \lim _{u \rightarrow u_{0}} f(u)=f\left(u_{0}\right)=A \quad$ there is

$$
\lim _{x \rightarrow x_{0}} f(g(x))=A
$$

It needs cautions that, because the primary functions are all continuous functions within it domain, so that, when discussion the ultimate value of primary compound functions, the discipline 3 can always be used for solving such problems, but this doesn't mean that all the ultimate value of compound functions can be solved by using discipline 3, the example 2 and 3 are both this kinds of situations, while we can not considerate that.

$$
\lim _{x \rightarrow 0} \ln (1+x)^{\frac{1}{x}}=\ln \left[\lim _{x \rightarrow 0}(1+x)^{\frac{1}{x}}\right]=\ln e=1
$$

is because of the continuality of $\ln u$,because $\ln u$ is the continuous function of $u$, $\lim _{u \rightarrow e} \ln u=\ln e$,but $\ln (1+x)^{\frac{1}{x}}$ and $g(x)=(1+x)^{\frac{1}{x}}$ are not continuous at $x=0$, so that the disciple 3 can not be applied here, especially it can be point out that, the inverse function is a ultimate value problems of compound functions.

$$
\begin{aligned}
& \text { Set } g(x)=\frac{\Delta y}{\Delta x}=\frac{f(x)-f\left(x_{0}\right)}{x \rightarrow x_{0}}, f(u)=\frac{1}{u}, \\
& \text { Obviously } u_{0} \neq 0 \text { there is } \lim _{u \rightarrow u_{0}} f(u)=\lim _{u \rightarrow u_{0}} \frac{1}{u}=\frac{1}{u_{0}},
\end{aligned}
$$

$\operatorname{But} \lim _{x \rightarrow x_{0}} g(x)=f^{\prime}\left(x_{0}\right)=u_{0}$, is there existing $\lim _{x \rightarrow x_{0}} f[g(x)]=\lim _{u \rightarrow u_{0}} f(u)=\frac{1}{u_{0}}$ is a problem.

so that, it must based on the conditions of getting inverse functions : first, the inverse function is existing ,then the mapping of its original functions must be one to one mapping,so that when $x \neq x_{0}$, $y \neq y_{0}$, Second, because $u_{0} \neq 0$, and $f\left(u_{0}\right)=\frac{1}{u_{0}}$ is existing,this exclude when $x \rightarrow x_{0}$, during $u \rightarrow u_{0}$ the range of $u$ make change the range value of $\lim _{u \rightarrow u_{0}} f(u)=A$, so based on rule 1 ,

\section{Reference}

$$
\lim _{\Delta x \rightarrow 0} \frac{\Delta y}{\Delta x}=\frac{1}{\lim _{\Delta y \rightarrow 0} \frac{\Delta x}{\Delta y}}=\frac{1}{x^{\prime}(y)} \quad x^{\prime}(y) \neq 0 .
$$

[1] Long Wang. <<Fundamental of Calculus $>>$ [M], Shanghai: Press of East China University of Science and Technology, 2015,2: 79-86

[2] Cuilian Feng, Shutian, Liu, $<<$ the solutions and assistant for Calculus Learning $>>$ [M], Beijing: Higher Education Press, 2006,12.

[3] Math Department of Beijing University, Higher Mathematics, Higher Education Press, 1998

[4] Hua Wuxiong, Matrix Analysis, Tongji University Press, 1993

[5] Da Xincheng, Matrix Analysis, Shanghai Jiaotong University, 1991 